\title{
ESTUDIO DE EVALUACIÓN EN HÁBITOS Y ESTILO DE VIDA SALUDABLE EN PERSONAS MAYORES DEL MUNICIPIO DE VILLA DEL ROSARIO.
}

\author{
Ortiz. S, Wilson ${ }^{1}$, Caicedo. P, Sergio Andrés ${ }^{2}$, Ortega. P, Arles Javier ${ }^{3}$ \\ ${ }^{1}$ Magister en Ciencias de la Actividad Física y el deporte. Docente programa Educación física recreación y deportes. Universidad \\ de Pamplona wos0000@hotmail.com \\ ${ }^{2}$ Magister en Ciencias de la Actividad Física y el deporte. Docente programa Educación física recreación y deportes. Universidad \\ de Pamplona. sergio.caicedo@unipamplona.edu.co \\ ${ }^{3}$ Doctor en Ciencias de la Cultura Física. Docente programa Educación física recreación y deportes. Universidad de Pamplona. \\ arlesjavierortegap@hotmail.com.
}

\section{RESUMEN}

Los beneficios para la salud de la actividad física han sido documentados en numerosos estudios. Estos comprenden la reducción del riesgo de morir por enfermedades cardiovasculares, el control del sobrepeso y la obesidad, disminución de la probabilidad de presentar diabetes mellitus tipo 2 (diabetes del adulto) y osteoporosis (descalcificación de los huesos); además de la reducción del riesgo de padecer de algunos tipos de cáncer (colón, próstata, seno, pulmón y endometrio); así como disminución de la ansiedad, la depresión y reducción del estrés. Guía comunitaria de Actividad Física (2004). En la presente investigación se expone el estudio realizado con una población conformada por un grupo de treinta (10) personas mayores comprendidas entre 60 y 89 años integrantes de la asociación Adulto Mayor García Herreros del municipio Villa del Rosario, a quienes se le desarrolló el estudio en tres momentos, la fase I, consistió en la aplicabilidad de un cuestionario internacional para la actividad física (IPAQ), por Duperly J. y et., (2011), y la realización de los test de valoración de la condición física de los adultos mayores a través de la batería SENIOR FITNES TEST (FST) diseñada por (Rikli y Jones 2001), la fase ॥ denominada de intervención desarrollado a través de las sesiones de actividad física dirigida musicalizada, de manera regular, cumpliendo con las recomendaciones de la OMS (2010) por Duperly J. y et., (2011), y la fase III, de evaluación se observan los resultados obtenidos indicando el estilo de vida y la aptitud física de las personas mayores objeto de estudio y su evolución durante el proceso de investigación.

PALABRAS CLAVES: personas mayores, actividad física, aptitud física.

\begin{abstract}
The health benefits of physical activity have been documented in numerous estudies. These include reducing the risk of dying from cardiovascular disease, the management of overweight and obesity, decreased likelihood of developing diabetes mellitus type 2 (adult onset diabetes) and osteoporosis (softening of the bones); in addition to reducing the risk of some cancers (colon, prostate, breast, lung and endometrial cancer); and decreased anxiety, depression and stress reduction. In addition, physical activity performed properly, can rehabilitate patients with cardiovascular disease; It is used as an assistant to treat many diseases that affect the joints, bones, muscles and the defense system. The effects of physical activity can improve the work environment, the ability to concentrate; They give quality of life and independence in the elderly of Community Physical Activity Guide (2004). In the present research the study was carried out with a population formed by a group of thirty (10) elderly people between 60 and 89 years old members of the association Elderly Garcia Herreros of the municipality Villa del Rosario, to whom the study was developed In three stages, phase I consisted of the applicability of an international questionnaire for physical activity (IPAQ) by Duperly J. et al. (2011), and the performance of the physical fitness assessment Older adults through the SENIOR FITNESS TEST (FST) battery designed by (Rikli and Jones 2001), Phase II called intervention developed through sessions of music-directed physical activity, on a regular basis, complying with the recommendations of the (2010) by Duperly J. et al., (2011), and phase III, evaluation are observed the results obtained indicating the lifestyle and physical fitness of the elderly subject of study and their evolution during the process research.
\end{abstract}

KEY WORDS: Elderly - physical activity - physical fitness.

Convocatoria III. Trimestre. Recibido el 15 de junio de 2017; Aceptado el 15 de julio de 2017. 


\section{INTRODUCCIÓN}

El impacto global de la Actividad física en la intervención, el manejo y la rehabilitación de las ECNT, ha sido bien documentado en las últimas décadas. Se han demostrado beneficios claros en la prevención y manejo de la enfermedad cardiovascular, la diabetes tipo 2, el sobrepeso y la obesidad, el cáncer de colon, seno y próstata, la osteoporosis así como también de algunas enfermedades mentales $y$ neurodegenerativas. Las guías internacionales basadas en numerosos estudios, recomiendan por lo menos 150 minutos a la semana de Actividad Física moderada o vigorosa para toda la población, siendo mayores los beneficios si se alcanzan más de 300 minutos a la semana en especial para niños y adultos con sobrepeso, adicionalmente se recomienda el entrenamiento de la fuerza muscular por lo menos dos veces a la semana en especial en adultos mayores cuya pérdida de masa muscular es frecuente $y$ perjudicial. Se debe tener en cuenta la edad, los factores de riesgo cardiovascular (hipertensión arterial, dislipidemia, diabetes mellitus tipo 2, tabaquismo, sedentarismo y otros), las condiciones musculo- esqueléticas de las personas, los gustos e intereses, el grado de motivación 0 estadio de cambio comportamental, los horarios y las posibilidades de acceso a espacios o personal calificado, entre otros. Se deben incluir recomendaciones específicas de frecuencia, intensidad, tiempo y tipo de ejercicio. Duperly J. y et., (2011).

En las Asociaciones del Adulto Mayor del municipio de Villa del Rosario, se evidencia un bajo nivel en cuanto a la práctica regular de Actividad física, debido a diferentes factores, entre ellos se encuentra la falta de recursos económicos, el desconocimiento por parte de los líderes de estas de las asociaciones, con respecto a los beneficios que se obtienen cumpliendo con las recomendaciones de Actividad física en el Adulto Mayor, y el poco interés de la Alcaldía municipal y los diferentes entes como desarrollo comunitario y el IMRD municipal con respecto al apoyo de profesionales que intervengan los procesos en estos grupos poblacionales.

por lo anterior en la Asociación del Adulto Mayor García Herreros del municipio de Villa del Rosario, se identificó a través del Cuestionario internacional para la actividad física (IPAQ), por Duperly J. y et., (2011), el nivel de actividad física, el estado nutricional y el consumo de tabaco, en la población objeto de estudio teniendo en cuenta las recomendaciones de la Organización Mundial de la Salud (2010), por Duperly J. y et., (2011), Además se realizó la aplicación de una serie de pruebas que buscan valorar la condición física de los adultos mayores a través de la batería SENIOR FITNES TEST (FST) diseñada por (Rikli y Jones 2001), con lo cual se medirá con éxito el nivel actual de aptitud física de los adultos mayores en el municipio de Villa del Rosario.

\section{Fundamentación Teórica.}

De acuerdo al análisis documental realizado y a los objetivos planteados se referencia algunas investigaciones a nivel internacional, nacional y regional que aportan al objeto de estudio del presente trabajo investigativo.

A nivel Internacional se evidencia el trabajo realizado por García, y et. (1996), presentan el último capítulo de su libro, sobre pruebas para la valoración motriz con los resultados obtenidos de un grupo de 1845 sujetos de la isla gran canaria, con edades entre 10 y 65 años.

De igual manera el siguiente estudio internacional es de tipo descriptivo, se dio por Navarro. (1997) en su tesis doctoral, a una muestra de 1111 sujetos donde valoró el nivel de condición física.

Sobre la base de los argumentos y criterios anteriormente expuestos se ha llegado a precisar el siguiente problema científico:

¿Qué particularidades determinan la Aptitud Física de las personas que asisten a la Ciclo vía del municipio Villa del Rosario?

\section{DESARROLLO METODOLÓGICO}

El tipo de investigación del presente estudio es descriptivo, el cual busca especificar las propiedades más importantes de personas, grupos, comunidades, entes u otro fenómeno que sea sometido a un proceso de análisis; como lo refiere. 
Méndez (2003), indica que la investigación descriptiva utiliza criterios sistemáticos que permiten poner de manifiesto la estructura de los fenómenos en estudio, además ayuda a establecer comportamientos concretos mediante el manejo de técnicas específicas de recolección de información. Este tipo de investigación permitirá al presente estudio realizar una valoración de la aptitud física en los asistentes de la ciclo vía del municipio Villa del Rosario." y de esta manera generar un cambio en la aptitud de las personas buscando aumentar la prevalencia de la Actividad física en la población objeto de estudio.

Para la investigación se tiene en cuenta el consentimiento informado ya que se realizó con seres humanos, como lo menciona el Tribunal de Nuremberg que evidenció la necesidad del consentimiento informado de la siguiente manera Código de Nuremberg (1947) cuya primer artículo dice: "El consentimiento voluntario del sujeto humano es absolutamente esencial. Esto quiere decir que la persona involucrada debe tener capacidad legal para dar su consentimiento; debe estar situada de tal forma que le permita ejercer su libertad de escoger, sin la intervención de cualquier otro elemento de fuerza, fraude, engaño, coacción 0 algún otro factor posterior para obligarlo a coercer, y debe tener el suficiente conocimiento y comprensión de los elementos para permitirle tomar una decisión correcta".

\section{FASES DEL ESTUDIO}

\section{Tabla N 1}

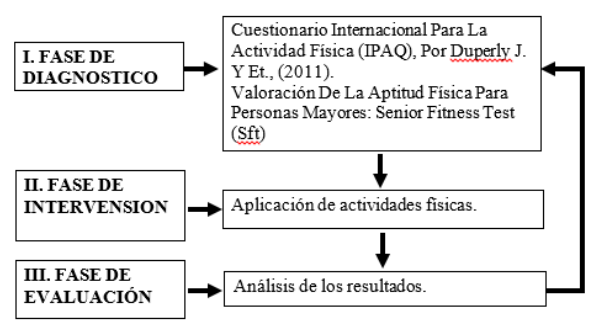

Así mismo en la investigación se tiene en cuenta los principios éticos definidos como los tres principios básicos del informe Belmont (1979) se enfocan en el respeto por las personas, beneficencia y justicia; y se han establecido así porque se consideran como valores morales que rigen a la sociedad y ello tiene que ver con las conductas propias de cada individuo.

\section{POBLACIÓN Y MUESTRA POBLACIÓN}

Tamayo y et., al 1997. "La población se define como la totalidad del fenómeno a estudiar donde las unidades de población posee una característica común la cual se estudia y da origen a los datos de la investigación" (p. 114). En esta investigación se tomó una población de 120 personas mayores de la Asociación del Adulto Mayor García Herreros del municipio de Villa Del Rosario, los cuales fueron filtrados por criterio de inclusión y exclusión según los códigos de ética avalados por: Helsinki, Núremberg y Belmont para llegar a una muestra.

\section{MUESTRA}

Según Tamayo, T Tamayo y et., al 1997. Afirma que la muestra es "el grupo de individuos que se toma de la población, para estudiar un fenómeno estadístico" (p. 38).

Por lo que la muestra esta con formada por 10 personas mayores que integrantes de la "Asociación del Adulto mayor García Herreros en edades comprendidas entre 60 y 89 años municipio Villa del Rosario"; los cuales fueren seleccionados por criterios de inclusión y exclusión según los códigos de ética en investigación con seres humanos.

\section{INSTRUMENTOS}

- Cuestionario Internacional Para La Actividad Física (IPAQ), Por Duperly J. Y Et., (2011).

- Valoración De La Aptitud Física Para Personas Mayores: Senior Fitness Test (Sft)

- Prueba de sentarse y levantarse de una silla

- Prueba de flexiones del brazo

- Prueba de caminata de 6 minutos

- Prueba de flexión del tronco en silla 
- Prueba de juntar las manos detrás de la espalda

- Prueba de levantarse, caminar y volverse a sentar

\section{MÉTODOS Y TÉCNICAS}

En cuanto al método y técnicas, este trabajo se desarrolló en tres fases, observar Tabla N 1; los cuales fueron aplicados en la Asociación del Adulto mayor García Herreros del Municipio de villa del Rosario de la siguiente manera: Fase I, consistió en la aplicación a manera de pre test y pos test del Cuestionario internacional para la actividad física (IPAQ), (2011), la cual contiene antecedentes personales sobre el nivel de actividad física, el estado nutricional y el consumo de tabaco, morbilidad reportada donde se conocerá su estado de salud actual y las mediciones directas, donde se tomó la talla el peso y el perímetro de cintura; a su vez la aplicación a manera de pre test y pos test de una serie de pruebas que buscan valorar la condición física de los adultos mayores a través de la batería Senior Fitnes Test (FST), diseñada por Rikli y Jones, (2001), siendo esta una herramienta que nos permitiese valorar la condición física de los mayores con seguridad así como de forma práctica, la fase ॥ denominada de intervención desarrollado a través de las sesiones de actividad física dirigida musicalizada, de manera regular, cumpliendo con las recomendaciones de la OMS (2010) por Duperly J. y et., (2011), y la fase III, de evaluación se observan los resultados obtenidos indicando el estilo de vida y la aptitud física de las personas mayores objeto de estudio y su evolución durante el proceso de investigación. Es importante destacar que se recomienda que realice actividad física por lo menos 150 minutos a la semana, consumir 5 porciones entre frutas y verduras al día y proteger espacios $100 \%$ libres de humo de tabaco.

\section{PAQUETE ESTADÍSTICO}

A partir de los datos obtenidos de las observaciones realizadas, y las pruebas aplicadas en el estudio, se confeccionó una base de datos para su procesamiento, con el apoyo del paquete estadístico SPSS 20 , con el fin de analizar los resultados.

\section{RESULTADOS}

Fase I. La aplicación del cuestionario internacional para la actividad física (IPAQ) por Duperly J. y et., (2011), Ver grafica 1 y 2 se aplicó de forma individual a 10 Personas mayores de la Asociación del Adulto Mayor García Herreros del municipio de Villa del Rosario, donde se empleó un formato en físico del cuestionario, guiado por un encuestador.

¿Cuántos días practica actividad física de intensidad moderada en su tiempo libre?

Gráfica N 1

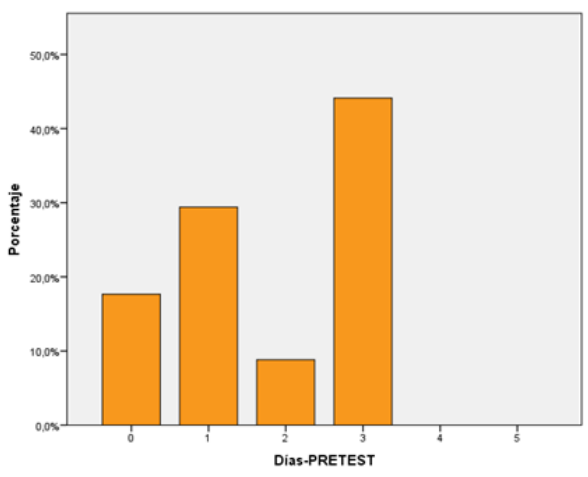

\section{Gráfica N 2}

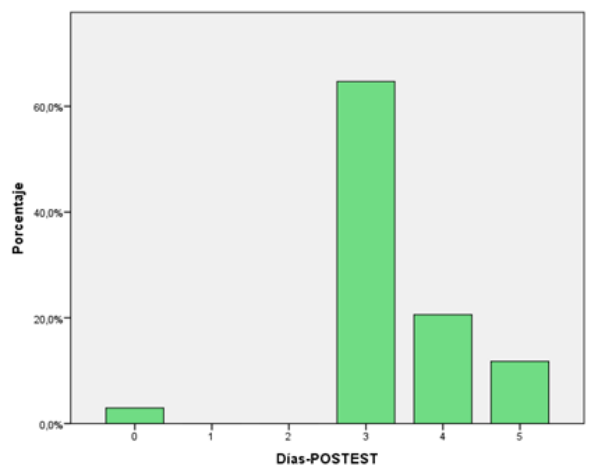

De las 10 personas encuestadas se puede identificar que: 
En el test inicial solo el 3 personas representadas con el $44 \%$ de la población estudiada realiza Actividad física de manera regular más de 3 veces por semana, en el test final, luego de realizar la intervención este porcentaje aumenta siendo el 7 personas con el $97 \%$ de la población quienes realizan actividad física de manera regular, cumpliendo con las recomendaciones de la OMS.

En esta fase se aplicó la batería Senior Fitnes Test (FST) diseñada por (Rikli y Jones 2001), a manera de pre test y pos test, con lo cual se medirá con éxito el nivel actual de aptitud física de los adultos mayores en el municipio de Villa del Rosario.

Tabla N 2. Recolección de los datos de pretest y postest.
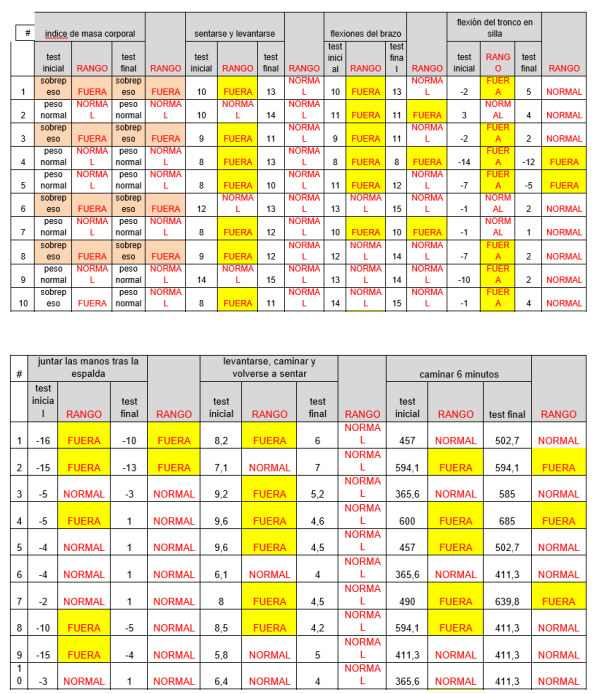

Como se puede apreciar en la tabla N 2 se presentan a modo de pre test y pos test, los resultados obtenidos en la presente investigación, logrando conocer los hábitos y estilos de vida de las personas mayores de la asociación del adulto mayor García Herreros del municipio de Villa del Rosario.

Prueba Indice De Masa Corporal (Imc).

\section{Gráfica N 3}

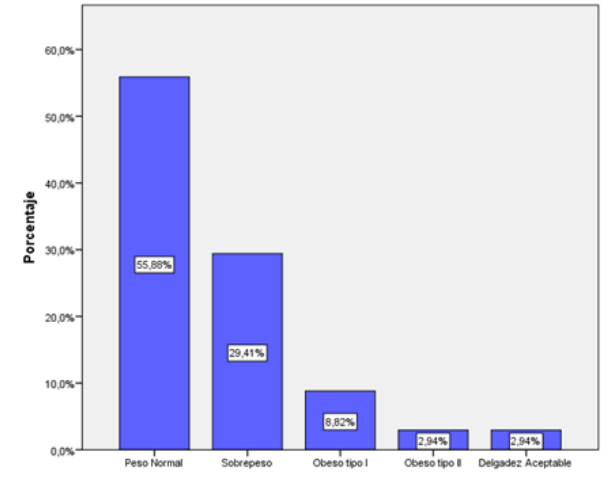

Gráfica N 4

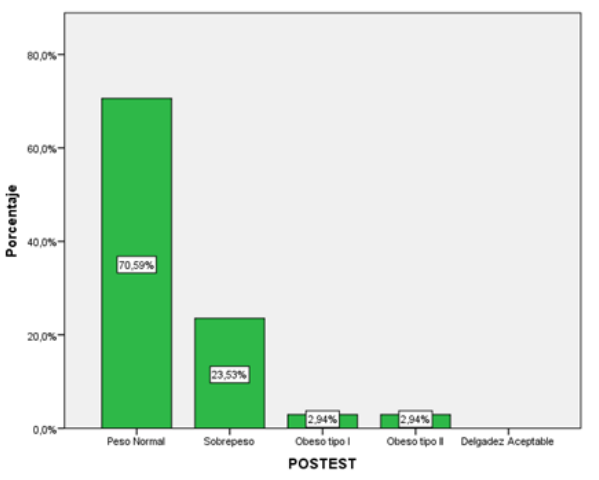

Análisis de las gráficas 3 y 4 :

- Las personas con un Peso Normal aumentaron, pasando de un $55,88 \%$ a un $70,59 \%$.

- Las personas con sobrepeso disminuyeron, pasando de un $29,41 \%$ a un $23,53 \%$.

Las personas con obesidad tipo I disminuyeron, pasando de un $8,82 \%$ a un $2,94 \%$.

Las personas con obesidad tipo ii se mantuvieron en un $2,94 \%$.

- $\quad$ Las personas con delgadez aceptable disminuyeron, pasando de un $2,94 \%$ a un $0 \%$.

\section{CONCLUSIONES}

El $44 \%$ de la población estudiada realiza Actividad física de manera regular más de 3 veces por semana, en el test inicial, luego de realizar la intervención este porcentaje aumenta al el $97 \%$ de la población en el test final, cumpliendo con las recomendaciones de la OMS. 
Resultados obtenidos de la aplicación de la batería SENIOR FITNES TEST (FST) diseñada por (Rikli y Jones 2002), a manera de pre test y pos test, con lo cual se midió con éxito el nivel actual de aptitud física de los adultos mayores en el municipio de Villa del Rosario.

Al analizar el test inicial y final de sentarse y levantarse de una silla se encontró que las personas valoradas dentro de un rango NORMAL representaban un $44,12 \%$, con la aplicación del proyecto se logró aumentar a un $88,24 \%$; mientras que las personas que se sentaban y levantaban de una silla valoradas dentro de un rango por FUERA de lo normal representaban un $55,88 \%$, con la aplicación del proyecto se logró disminuirlo a un $11,76 \%$.

En el test de caminata durante 6 minutos continuos, las personas valoradas dentro de un rango NORMAL representaban un $41,18 \%$ en el test inicial, con la aplicación del proyecto se logró aumentar a un $79,41 \%$ en el test final.

\section{REFERENCIAS BIBLIOGRÁFICAS}

Arias Herrera, Héctor (1995). La comunidad y su estudio. Personalidad, educación, salud. Ciudad Habana. Editorial Pueblo y Educación. 97 p.

Barbero, J. M. y Cortés, F. (2005) Trabajo Comunitario, organización y desarrollo social. Madrid: Alianza Editorial.

Burnham, J.M. (1998).Exercise is medicine: health benefits of regular Physical activity. J-la State -Med-Soc. 1998 Jul; 150 (7). 319-23.

Belmont (1979). Informe Belmont Principios Éticos y Directrices para la Protección de Sujetos

Bennassar M. (2011). Estilos de vida y salud en estudiantes universitarios: La universidad como entorno promotor de la salud. Universitat de les Illes Balears. España. Recuperado de: http://www.academia.edu/6223906/Estilos_de_vi da_y_salud_en_estudiantes_universitarios_la_u niversidad_como_entorno_promotor_de_la_salu d._Miguel_Bennasar_Veny.

Coldeportes (2011). "Hábitos y estilos de vida saludable". Tomo II

COLDEPORTES (2016). IV Curso de Actividad Física Dirigida Musicalizada. Recuperado de: http://www.coldeportes.gov.co/fomento_desarrol lo/actividad_fisica/sistema_nacional_capacitacio n/iv_curso_nacional_actividad_fisica_81858_81 858

Casimiro, A.J. (2000). Educación para la salud, actividad física y estilo de vida. Universidad de Almería. Servicio de Publicaciones.

Cooper, D.M. (1994). Evidence for and mechanisms of exercise modulation of growth: an overview. Medicine and science in sports and exercise; 26,6:733-740.

Corbin, C.B. (1987). Youth fitness, execise and health: There is much to be done. Research Quartely for Exercise and Sport. 58, 4, 308-14.

Del Sol, F. (2000). Evaluación de un programa adaptado de educación física en niños y niñas de siete años en el ámbito de la salud. Tesis Doctoral. Universidad de Granada.

Devis, J. y Peiró, C. (1992). Nuevas perspectivas curriculares en educación Física: la salud y los juegos modificados. Barcelona: INDE.

Espinoza L. y et., al (2011). Hábitos de alimentación y actividad física en estudiantes universitarios. Valparaíso, Chile. Recuperado de:

http://www.scielo.cl/pdf/rchnut/v38n4/art09.pdf

Guerrero L. y et., al (2009). Estilo de vida y salud. Merida, Venezuela. Recuperado de: http://www.saber.ula.ve/bitstream/123456789/32 226/1/articulo1.pdf 
Lozano O. y et., al (2014). Lineamientos y oportunidades de Mejora en el Desarrollo de la Acción de Gestores, Monitores y Lideres del Programa de Hábitos y Estilos de Vida Saludable. Coldeportes. Recuperado de:

http://www.coldeportes.gov.co/recursos_user/we b_coldeportes/2014/lineamientos-

oportunidades-web.pdf

Núremberg, (1947) Código de Núremberg. Principios de ética, bioética y conocimiento del hombre. Alemania. Recuperado de:http://www.uaeh.edu.mx/investigacion/produc tos/4822/libro_principios_de_etica.pdf

Organización Mundial de la Salud. Relaciones entre los programas de salud y el desarrollo social y económico. Ginebra: OMS; 1968. Recuperado

de: https://digitum.um.es/jspui/bitstream/10201/6621 /1/CONDICI\%C3\%93N\%20F\%C3\%8DSICA \%20 Y\%20SALUD.pdf

Organización Mundial De La Salud (2016). Estrategia mundial sobre régimen alimentario, actividad física y salud.

Posada L. (2013) Educación Física Juvenil I.E.D. - localidad séptima de Bogotá. Recuperado de: http://edufisicayrecreacion.blogspot.com.co/p/te st-fisicos.html

Tamayo y Tamayo, Mario. (1997) El Proceso de la Investigación científica. Editorial Limusa S.A. México.

Guerra Alfonso, Alaine (1999) Diseño de un programa de actividades deportivas recreativas para una comunidad rural. Trabajo de diploma Sancti Spíritus.

Rodríguez, F. A. (1995). Prescripción de ejercicio para la salud (I) Resistencia Cardiorrespiratoria. Apunts: Educación Física y Deportes 1995(39)

8710 
